\title{
Radiotherapy in small cell cancer of the lung treated with combination chemotherapy: a controlled trial
}

\author{
R L SOUHAMI, D M GEDDES, S G SPIRO, P G HARPER, J S TOBIAS, B S MANTELL, \\ F FEARON, I BRADBURY
}

\begin{abstract}
Three hundred and seventy one patients with extensive or limited small cell cancer of the lung took part in a randomised trial to evaluate irradiation of the primary tumour in patients treated with combination chemotherapy. They were randomly allocated to treatment either with chemotherapy alone (198) or with both chemotherapy and radiotherapy (173). All 371 initially received 12 weeks of chemotherapy after which they were categorised according to response. Only those with stable or responding disease continued with their allotted treatment. Thus 144 received chemotherapy alone and 135 received both chemotherapy and radiotherapy. The design of the study permitted analysis of the effect of radiotherapy according to initial extent of disease and quality of early response to chemotherapy.

Radiotherapy (40 Gy (4000 rad), one fraction a day over 20 days) did not improve the survival of patients in any category of initial extent of disease or early response to chemotherapy and was not effective in preventing local relapse.
\end{abstract}

\footnotetext{
Department of Radiotherapy and Oncology, University College Hospital, London WC1

R L SOUHAMI, MD, FRCP, consultant physician

I S TOBIAS, FRCR, MRCP, consultant in radiotherapy and oncology F FEARON, SRN, research nurse

I BRADBURY, BSC, research assistant

P G HARPER, MRCP, senior registrar

London Chest Hospital, London E2

D M GEDDES, MD, FRCP, consultant physician

B S MANTELL, FRCR, MRCP, consultant in radiotherapy and oncology

Brompton Hospital, London SW3

S G SPIRO, MD, FRCP, consultant physician

Correspondence to: Dr R L Souhami.
}

\section{Introduction}

Small cell cancer of the lung is sensitive to cytotoxic drugs and also to radiotherapy. Randomised trials have shown that the addition of combination chemotherapy to irradiation of the primary tumour may appreciably improve survival compared with radiotherapy alone ${ }^{1}$; similarly, as relapse in the lung is common in patients treated with chemotherapy alone, irradiation of the primary site is often given as part of treatment. The contribution of radiotherapy to survival in patients treated with chemotherapy is, however, uncertain. ${ }^{23}$ We report the results of a prospective randomised trial of the efficacy of radiotherapy in patients with small cell cancer of the lung treated with chemotherapy.

\section{Patients and methods}

From June 1979 to January 1982, 380 patients with small cell cancer of the lung were entered into this study. Patients were excluded if they were over 75 years of age or had another malignancy or disease that made chemotherapy impossible. Obstruction of the superior vena cava was not a criterion for exclusion. Diagnosis was made either on the basis of histological findings obtained at bronchoscopy or biopsy of another site, or on the basis of unequivocal cytological features. Informed consent was obtained from each patient, and on entry into the study they were randomly allotted, using a sealed envelope system, to receive either chemotherapy alone or chemotherapy and radiotherapy. Of the 380 patients entered into the study, 371 could be evaluated (table I); the nine others were excluded because of major violations of protocol in two cases, incorrect diagnosis in one, lack of follow up information in one, and early withdrawal from the trial in five. Once entered, all the remaining patients were considered to be evaluable regardless of the number of cycles of chemotherapy they received. Before treatment all patients underwent chest $x$ ray examination, liver function tests, and isotope bone and liver scanning. Patients were categorised as having limited disease (disease confined to one hemothorax and ipsilateral supraclavicular nodes) or extensive disease (a wider spread of tumour within the thorax or distant metastases). Randomisation to treatment was not stratified according to extent of disease.

Chemotherapy consisted of doxorubicin $50 \mathrm{mg} / \mathrm{m}^{2}$ and vincristine $1.4 \mathrm{mg} / \mathrm{m}^{2}$ (maximum $2 \mathrm{mg}$ ) alternating every three weeks with cyclophosphamide $1 \mathrm{~g} / \mathrm{m}^{2}$ and methotrexate $50 \mathrm{mg} / \mathrm{m}^{2}$. All drugs were given intravenously through a fast running drip. A full blood count and 
chest radiography were done before each cycle of treatment and the dosages reduced if there was appreciable myelosuppression (total white cell count below $3.5 \times 10^{9} / 1$ or platelet count below $100 \times 10^{9} / 1$, or both). The response to chemotherapy was assessed after four cycles of treatment (two of each cycle). These responses to 12 weeks of treatment were categorised as complete, indicating disappearance of all evidence of disease on physical examination, chest radiography, and bronchoscopy; partial, indicating a reduction of more than half in the sum of the two maximum diameters at right angles on a chest radiograph; or non-existent, indicating any smaller response or progressive disease. Patients with responding or stable disease then received either eight further cycles of chemotherapy alone or radiotherapy (40 Gy (4000 rad) one fraction a day over 20 days) to the chest to encompass the primary and nodal sites of disease followed by eight

TABLE I-Details of 371 patients allotted to receive either chemotherapy alone or chemotherapy and radiotherapy

\begin{tabular}{lcc}
\hline & $\begin{array}{c}\text { Chemotherapy } \\
\text { alone } \\
(\mathrm{n}=198)\end{array}$ & $\begin{array}{c}\text { Chemotherapy } \\
\text { +radiotherapy } \\
(\mathrm{n}=173)\end{array}$ \\
\hline Median age (years) & 63 & 63 \\
No of men & 136 & 119 \\
No of women & 62 & 54 \\
No with limited disease & 73 & 57 \\
No with extensive disease & 125 & 116 \\
Performance score*: & 7 & 5 \\
$0-30$ & 59 & 54 \\
$40-60$ & 128 & 114 \\
$70-100$ & 4 & 3 \\
Not recorded & 4 & \\
\hline
\end{tabular}

*According to Karnofsky's scale.

cycles of chemotherapy. In all patients the sequence of cycles of chemotherapy was changed after the fourth cycle to avoid giving doxorubicin immediately after radiotherapy. No further chemotherapy was given to patients who had progressive disease, who were treated symptomatically, as were all patients who relapsed. Palliative treatment consisted of analgesics, steroids, and radiotherapy as clinically indicated for obstruction of the superior vena cava, painful bone metastases, and cerebral metastases. Prophylactic cranial irradiation was not given.

Life tables were analysed with the log rank test. The results are presented according to early response to chemotherapy and extent of disease. The effect of radiotherapy on local recurrence was assessed by plotting the cause specific cumulative hazard for local relapse. ${ }^{4}$

\section{Results}

Altogether 173 patients were randomly selected to receive both chemotherapy and radiotherapy. After four cycles of chemotherapy the disease was stable in $60(33 \%)$ and responding in $79(45 \%)$; all 139 went on to receive radiotherapy followed by chemotherapy. One hundred and ninety eight patients were randomly selected to receive chemotherapy alone. In $62(34 \%)$ the disease was stable and in $79(39 \%)$ it was responding after four cycles; chemotherapy was continued in these 141 . The early response to chemotherapy in all 371 patients was complete in 26 $(7 \%)$, partial in $132(35.6 \%)$, and stable disease or no response in $213(57 \cdot 4 \%)$. Figure 1 shows the survival of all patients according to their response at 12 weeks. The median survival for all 371 patients was 218 days. Response to chemotherapy was a major determinant of survival, the median survival in the 26 showing complete response being 427 days, in the 132 showing partial response 286 days, and in the 213 showing no response 147 days $(p<0.0001)$.

Patients treated with both chemotherapy and radiotherapy did not show any overall difference in survival compared with those treated with chemotherapy alone (fig 2), the median durations of survival after the start of treatment being 233 and 198 days respectively. The effect of radiotherapy was further analysed according to the initial extent of disease and early response to chemotherapy. Of 130 patients with limited disease, $65(50 \%)$ completely or partially responded to chemotherapy. Of these, 29 received radiotherapy and 36 did not. The median duration of survival in those receiving chemotherapy alone was 364 days; in those receiving combined chemotherapy and radiotherapy it was 398 days (fig 3). For the 65 patients with limited disease that did not respond the duration of survival in those receiving chemotherapy alone was 206 days and in those receiving chemotherapy

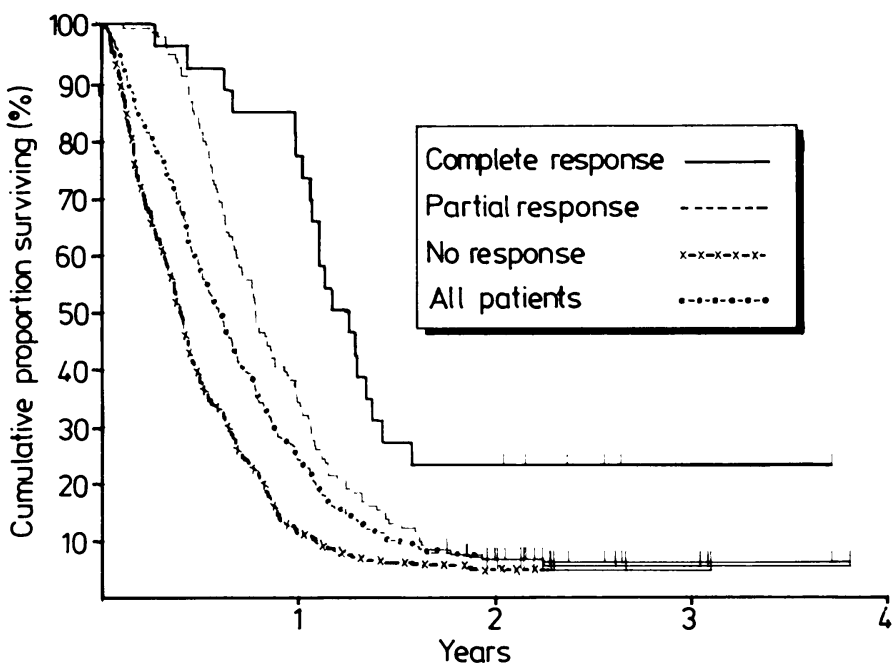

FIG 1-Survival in all patients and according to whether early response to chemotherapy was complete, partial, or non-existent.

and radiotherapy 243 day's (fig 4). Two hundred and forty one patients had had extensive disease at diagnosis. Of these, $93(38.6 \%)$ responded to chemotherapy and $148(61.4 \%)$ did not. In those who responded to chemotherapy the median survival in those given chemotherapy alone was 260 days and in those given chemotherapy and radiotherapy 256 days (fig 5). In patients with extensive disease that did not respond to chemotherapy those receiving chemotherapy alone survived for 96 days and those receiving combined treatment for 122 days (fig 6).

All patients were followed up for more than 21 months. Thirty $(8 \%)$ patients were alive at two years - that is, 16 months after treatment had stopped. Long term survivors did not predominate in the group who had received radiotherapy. Figure 7 shows the cumulative hazards of local and distant relapse. The slope of the curve represents the instantaneous probability of relapse, given that the patient has survived without relapse to a particular point in time. The plot suggests that the onset of local relapse may have been delayed in the patients who received chemotherapy and radiotherapy, but this did not reach significance. For relapse at distant sites the slopes of the lines were closely similar for patients receiving either treatment. Table II shows the incidence of relapse at different sites. Sixty four $(32.3 \%)$ of the patients allotted to receive chemotherapy alone and $48(27.7 \%)$ of those allotted to receive both chemotherapy and radiotherapy showed local relapse.

The side effects of chemotherapy were the same in the two groups. Almost all patients developed alopecia and drug induced nausea and vomiting of varying severity. Mucositis developed after treatment with

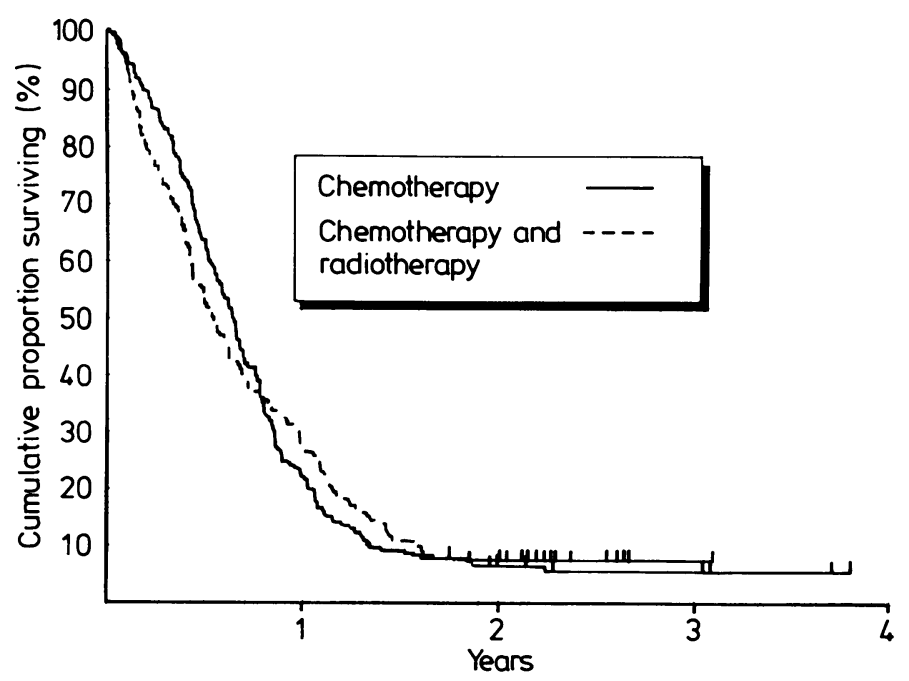

FIG 2-Survival in all 371 patients (responders and non-responders) according to whether they were allotted to receive chemotherapy alone $(n=198)$ or both chemotherapy and radiotherapy $(n=173)$. 


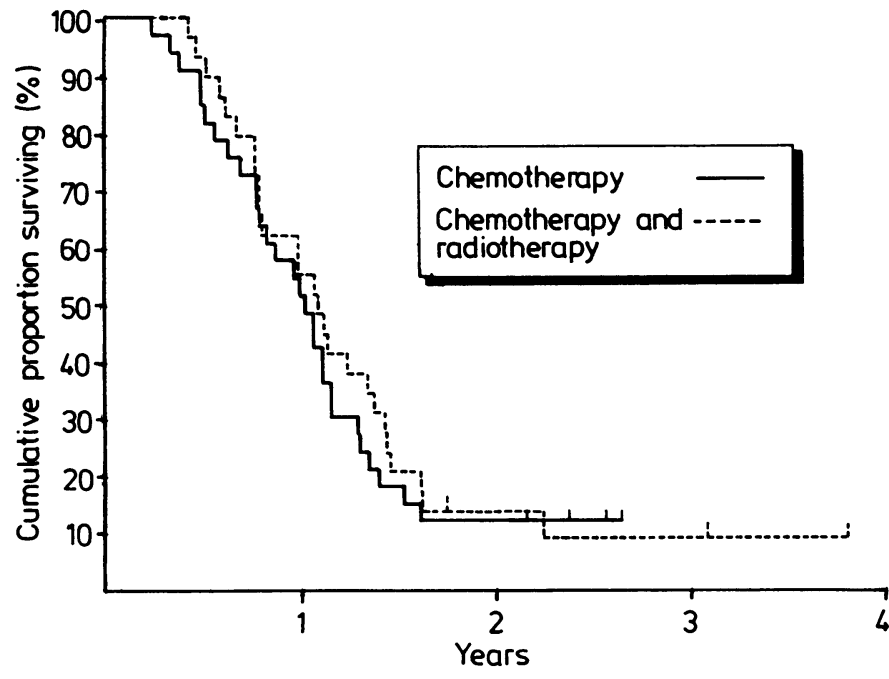

FIG 3-Survival in patients with limited disease that responded early to chemotherapy according to whether they received chemotherapy alone ( $\mathbf{n}=$ $36)$ or both chemotherapy and radiotherapy $(n=29)$.

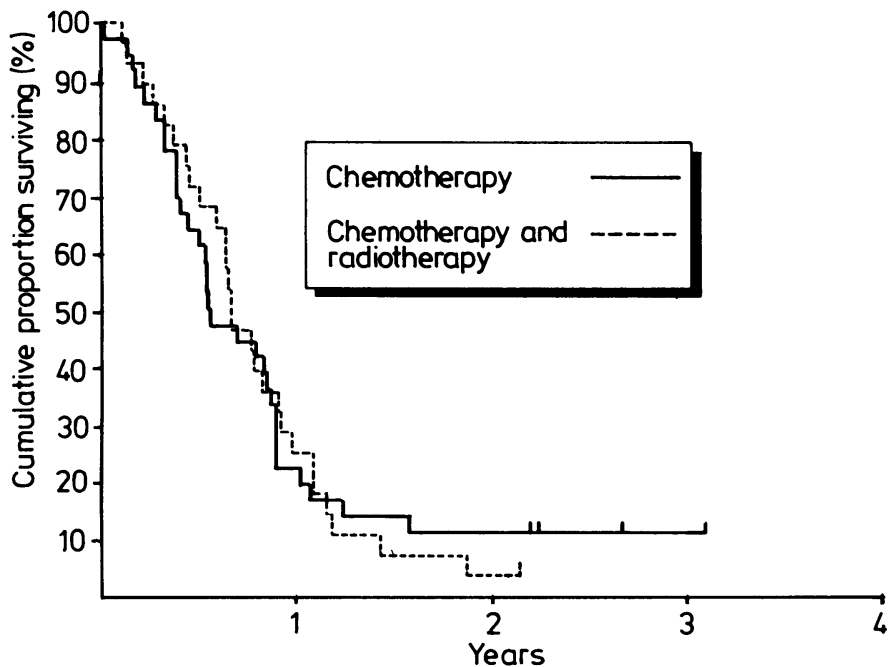

FIG 4-Survival in 65 patients with limited disease not responding to chemotherapy according to whether they received chemotherapy alone $(n=37)$ or chemotherapy and radiotherapy $(n=28)$.

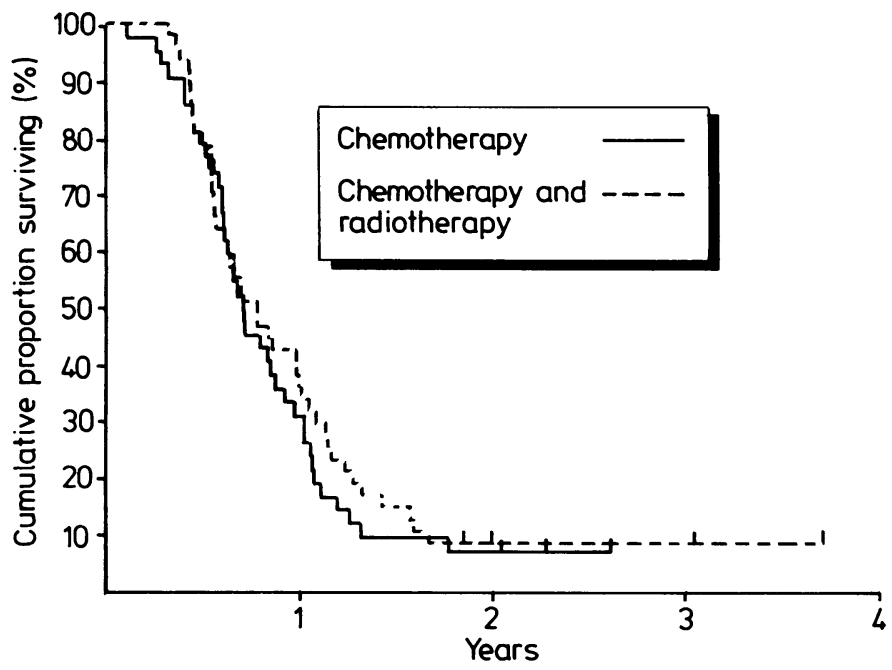

FIG 5-Survival in 93 patients with extensive disease responding to chemotherapy according to whether they received chemotherapy alone $(n=43)$ or chemotherapy and radiotherapy $(n=50)$.

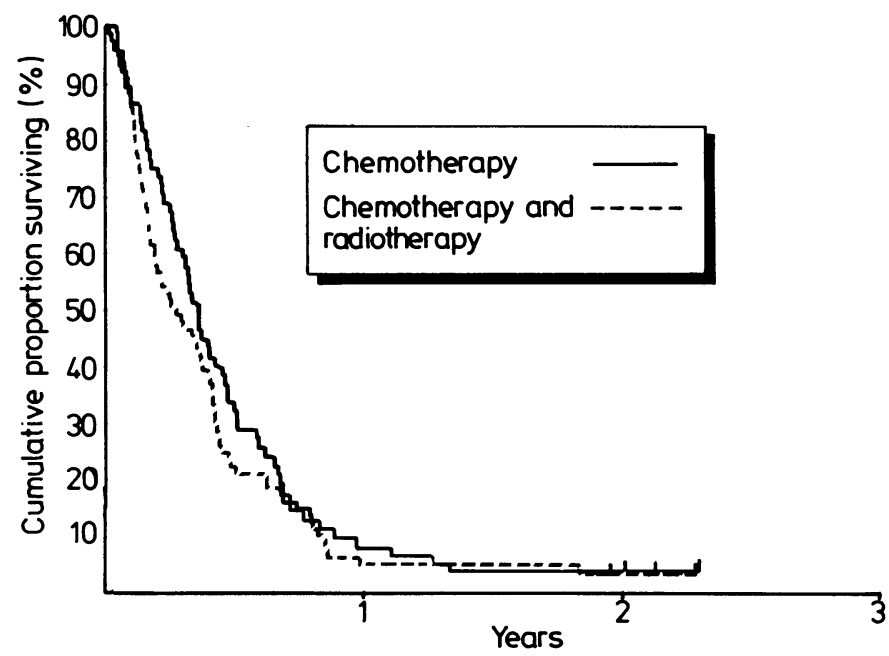

FIG 6-Survival in 148 patients with extensive disease not responding to chemotherapy according to whether they received chemotherapy alone ( $\mathbf{n}=$ $82)$ or chemotherapy and radiotherapy $(n=66)$.

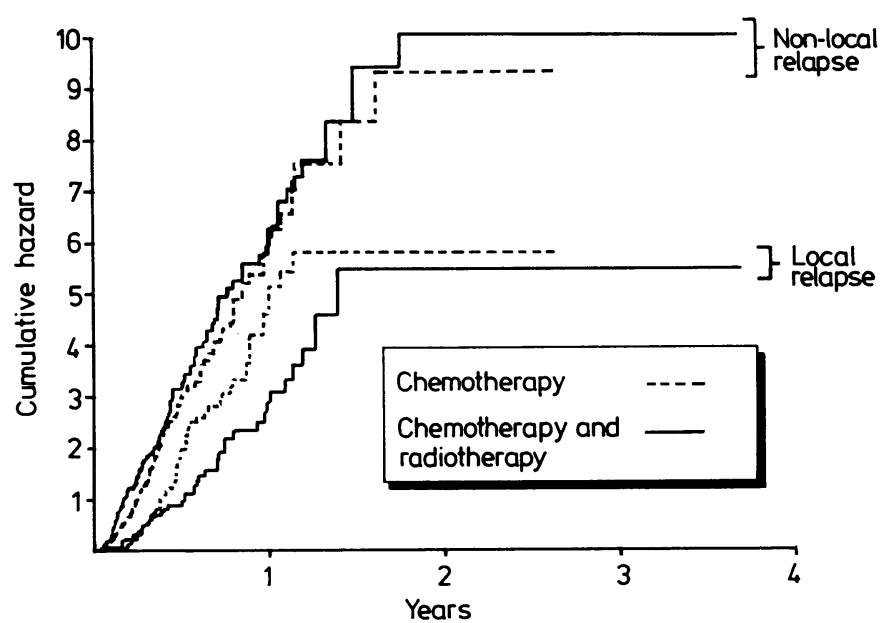

FIG 7-Cause specific hazards for local and non-local relapse in 198 patients allotted to receive chemotherapy alone and 173 allotted to receive both chemotherapy and radiotherapy.

TABLE II-Incidence of first relapse at different sites in patients allotted to receive either chemotherapy alone or chemotherapy and radiotherapy (figures are numbers (\%) of patients)

\begin{tabular}{lcc}
\hline & $\begin{array}{c}\text { Chemotherapy } \\
\text { alone }\end{array}$ & $\begin{array}{c}\text { Chemotherapy } \\
\text { +radiotherapy }\end{array}$ \\
\hline Intrathoracic (primary site) & $64(32 \cdot 3)$ & $48(27 \cdot 7)$ \\
Central nervous system & $25(12 \cdot 6)$ & $31(17 \cdot 9)$ \\
Liver & $8(4)$ & $17(9 \cdot 8)$ \\
Bone & $15(7 \cdot 6)$ & $12(6 \cdot 9)$ \\
Lymph nodes & $5(2 \cdot 5)$ & $6(3 \cdot 5)$ \\
Multiple & $68(34 \cdot 3)$ & $47(27)$ \\
Other & $2(1)$ & $3(1 \cdot 7)$ \\
No relapse & $11(5 \cdot 6)$ & $9(5 \cdot 2)$ \\
\hline Total & 198 & 173 \\
\hline
\end{tabular}

methotrexate in 74 patients $(20 \%$ ), who then received oral folinic acid ( $15 \mathrm{mg}$ every six hours for 24 hours) 24 hours after the methotrexate injection in subsequent cycles. There were four deaths related to treatment. These were due to infection resulting from bone marrow suppression, and two occurred in each treatment group. Additional side effects developed in patients receiving combined chemotherapy and radiotherapy, with transient anorexia and oesophagitis developing in most patients. Sixty one (35\%) developed radiological evidence of pulmonary fibrosis induced by radiation. Mild dyspnoea was attributable to this in $24(40 \%)$ of these cases. 


\section{Discussion}

Many studies have shown that response to chemotherapy is the major determinant of prognosis in small cell cancer of the lung, and this is confirmed by our study. The necessity of irradiation of the primary tumour is, however, the subject of controversy. ${ }^{23}$ In patients who are responding to chemotherapy thoracic irradiation might be expected to reduce the proportion of patients relapsing in the chest and result in improved survival. The efficacy of thoracic irradiation in producing local control is, however, a matter of dispute. McMahon et al suggested that a total dose of 40-45 Gy (4000-4500 rad) delayed local recurrence in comparison with $30 \mathrm{~Gy}$ (3000 rad), ${ }^{5}$ and Chalk et al reported that $50 \mathrm{~Gy}(5000 \mathrm{rad})$ over five weeks reduced the incidence of local failures in patients who responded completely to radiotherapy but not in others. ${ }^{6}$ Doses used to treat the tumour vary considerably but are usually about 40-45 Gy (4000-4500 rad) in daily fractions over 15-20 days. Cox et al suggested that a lower dose would be effective in patients treated with chemotherapy, but doses as high as $60 \mathrm{~Gy}$ (6000 rad) have been used by others. ${ }^{8}$ The definition of local relapse is itself problematical as the relapse may be intrathoracic yet just outside the area undergoing irradiation. ${ }^{9}$

Local relapse is therefore common after radiotherapy, and, though thoracic irradiation is often assumed to be necessary for long survival, its value can be assessed only by a randomised prospective trial.

The present study was larger and followed up its subjects for longer ( 21 months) than any previous study addressing this question. Previous studies have been small, ${ }^{10}$ restricted to patients with limited disease, ${ }^{11}$ or without long term follow up. ${ }^{12}{ }^{13}$ The design of our study permitted evaluation of radiotherapy in those patients who might have been expected to benefit from localised treatment-namely, those patients in whom the tumour was either responding to chemotherapy or not progressing. We included patients with both limited and extensive disease because those with extensive disease account for two thirds of all patients and the value of local radiotherapy had never been assessed in this group. We therefore analysed the effect of radiotherapy with respect to initial extent of disease and early response to chemotherapy.

The results show that there is no difference in survival between patients receiving chemotherapy alone and those receiving chemotherapy and radiotherapy in either limited or extensive disease. There is also no survival advantage in patients who respond to chemotherapy in either the short or the long term. The lack of effect of radiotherapy is confirmed by the failure to control local growth of tumours in patients receiving chemotherapy and radiotherapy. A non-significant delay in local relapse was observed in the patients treated with chemotherapy and radiotherapy. The dose administered (40 Gy $(4000 \mathrm{rad})$ in 20 daily fractions) is one that would be expected to be effective in small cell cancer of the lung and is widely used in the treatment of this tumour.

At the time of assessment of response to chemotherapy only a small proportion of patients $(7 \%$ ) showed a complete response, as judged by repeat bronchoscopy and chest radiography, and $35.6 \%$ showed a partial response. The low proportion of patients whose conditions responded is probably due to the early time of assessment of response ( 12 weeks after the start of treatment) and the relatively high proportion of patients (34\%) with a performance score of less than 70 . The many patients with relatively poor performance scores and with extensive disease are typical of those who present with the disease at district general hospitals, from which most of the patients were referred. The chemotherapy contained the most active agents known at the time the trial was started, but higher rates of response have recently been reported with regimens containing etoposide. ${ }^{14}$ Nevertheless, in the report of Aisner et al ${ }^{14}$ the proportion of all patients who survived beyond two years $(11.9 \%)$ was little greater than that in the present study, and it seems unlikely that our results would have been different if we had used other agents.
Early reports from other trials using different drugs and doses of radiation have also not shown any advantage for irradiation. In the randomised trial conducted by Fox et al chemotherapy comprised cyclophosphamide, doxorubicin, and vincristine and the dose of radiotherapy was the same. ${ }^{11}$ They did not treat patients with extensive disease. The trials conducted by Hansen et $a l^{12}$ and Stevens $e t a^{13}$ were similar in design, but different drugs were used and radiotherapy was given in two separate schedules of $20 \mathrm{~Gy}(2000 \mathrm{rad})$ and $17.5 \mathrm{~Gy}(1750 \mathrm{rad}) \mathrm{re}-$ spectively, each given over five days. Long term survival was not reported in these studies, but no survival advantage was seen with the addition of radiotherapy.

We therefore conclude that this dose of radiotherapy (40 Gy (4000 rad) to the intrathoracic tumour does not improve survival in patients with limited or extensive small cell cancer of the lung treated with chemotherapy who have stable or responding disease. This finding is in keeping with the early results of other randomised trials. Thoracic radiotherapy may, however, prove to be of value in patients whose tumours still show complete response at the end of chemotherapy, of whom a significant proportion may survive beyond two years. This question can only be answered by further trials.

This work was supported by the Cancer Research Campaign. The Research Centre for the Mathematical Modelling of Clinical Trials helped with the statistical analyses. We thank the many physicians and surgeons who participated in this study.

\section{References}

${ }^{1}$ Medical Research Council Lung Cancer Working Party. Radiotherapy alone or with chemotherapy in the treatment of small cell carcinoma of the lung. Br $\mathcal{F}$ Cancer 1979;40:1-9.

${ }^{2}$ Byhardt RW, Cox JD. Is chest radiotherapy necessary in any or all patients with small cell carcinoma of the lung ? Yes. Cancer Treat Rep 1983;67:209-15.

${ }^{3}$ Cohen $\mathrm{MH}$. Is thoracic radiation therapy necessary for patients with limited-stage small cell lung cancer? No. Cancer Treat Rep 1983;67: 217-21.

4 Kay R, Shumacher M. Unbiased assessment of treatment effects or disease recurrence and survival in clinical trials. Statistics in Medicine 1983;2: 41-58.

${ }^{5}$ McMahon LJ, Herman TS, Manning MR, Dean JC. Patterns of relapse in patients with small cell carcinoma of the lung treated with adriamycincyclophosphamide chemotherapy and radiation therapy. Cancer Treat Rep 1979;63:359-62.

6 Chalk LY, Daniels JR, Sikic BI, Torti FM, Lockbaum P, Carter SK. Patterns of failure in small cell carcinoma of the lung. Cancer 1982 50:1857-63.

${ }^{7}$ Cox JD, Byhardt R, Komaki R, Wilson JF, Libnoch JA, Hansen $\mathbf{R}$. Interaction of thoracic irradiation and chemotherapy on local control and survival in small cell carcinoma of the lung. Cancer Treat Rep 1979; $63: 1251-5$.

${ }^{8}$ Holoye PY, Samuels ML, Lanzotti VJ, Smith T, Barkely JT. Combination chemotherapy and radiation therapy for small cell carcinoma. $\mathcal{F} A M A$ $1977 ; 237: 1221-4$.

${ }^{9}$ Mira JG, Livinston RB. Evaluation and radiotherapy implications of chest relapse patterns in small cell lung carcinoma treated with radiotherapychemotherapy. Cancer $1980 ; 46: 2557-65$.

10 Williams C, Alexander M, Glatstein EJ, Daniels JR. Role of radiation therapy in combination with chemotherapy in extensive oat cell cancer of the lung: a randomised study. Cancer 1977;61:1427-31.

11 Fox RN, Woods RL, Brodie GN, Tattersall MH. A randomised study: small cell anaplastic lung cancer treated by combination chemotherapy and adjuvant radiotherapy. Int $\mathcal{F}$ Radiat Oncol Biol Phys 1980;6:1083-5.

12 Hansen HH, Dombernowsky P, Hansen HS, Rorth M. Chemotherapy versus chemotherapy plus radiotherapy in regional small cell carcinoma of the lung - a randomised trial. Proceedings of the American Association for Cancer Research 1979;20:277.

13 Stevens E, Einhorn L, Rohn R. Treatment of limited small cell lung cancer. Proceedings of the American Association for Cancer Research 1979;20: 435.

14 Aisner J, Whitacre M, Van Echo DA, Wiernik PH. Combination chemotherapy for small cell carcinoma of the lung: continuous versus alternating non-cross-reactive combinations. Cancer Treat Rep 1982;66: 221-30. 